Modulation spectroscopy study of the effects of growth interruptions on the interfaces of $\mathrm{GaAsSb} / \mathrm{GaAs}$ multiple quantum wells

This article has been downloaded from IOPscience. Please scroll down to see the full text article. 2006 J. Phys.: Condens. Matter 185927

(http://iopscience.iop.org/0953-8984/18/26/012)

The Table of Contents and more related content is available

Download details:

IP Address: 140.112.113.225

The article was downloaded on 11/02/2009 at 06:10

Please note that terms and conditions apply. 


\title{
Modulation spectroscopy study of the effects of growth interruptions on the interfaces of GaAsSb/GaAs multiple quantum wells
}

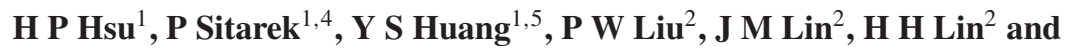 \\ K K Tiong ${ }^{3}$ \\ ${ }^{1}$ Department of Electronic Engineering, National Taiwan University of Science and Technology, \\ Taipei 106, Taiwan \\ ${ }^{2}$ Department of Electrical Engineering, National Taiwan University, Taipei 106, Taiwan \\ ${ }^{3}$ Department of Electrical Engineering, National Taiwan Ocean University, Keelung 202, Taiwan \\ E-mail: ysh@et.ntust.edu.tw
}

Received 3 March 2006

Published 16 June 2006

Online at stacks.iop.org/JPhysCM/18/5927

\begin{abstract}
The effects of growth interruption times combined with $\mathrm{Sb}$ exposure of $\mathrm{GaAsSb} / \mathrm{GaAs}$ multiple quantum wells (MQWs) have been investigated by using phototransmittance (PT), contactless electroreflectance (CER) and wavelength modulated surface photovoltage spectroscopy (WMSPS). The features originated from different portions of the samples, including interband transitions of MQWs, interfaces and GaAs, are observed and identified through a detailed comparison of the obtained spectra and theoretical calculation. A red-shift of the interband transitions and a broader lineshape of the fundamental transition are observed from samples grown under $\mathrm{Sb}$ exposure compared to the reference sample grown without interruption. The results can be interpreted in terms of both increases in $\mathrm{Sb}$ content and mixing of $\mathrm{Sb}$ in the GaAs interface layers. An additional feature has been observed below the GaAs region in the samples with $\mathrm{Sb}$ treatment. The probable origin of this additional feature is discussed.
\end{abstract}

\section{Introduction}

The GaAsSb/GaAs heterostructure system has attracted much attention recently because of its potential application in the semiconductor industry. In particular its type-II band alignment provides an excellent opportunity to improve the performance of both heterojunction bipolar transistors (HBTs) [1] and optoelectronic devices [2-4]. The defining feature of a type-II

4 Present address: Institute of Physics, Wrocław University of Technology, Wyb. St. Wyspianskiego 27, 50-370 Wrocław, Poland.

5 Author to whom any correspondence should be addressed. 
quantum well is the spatial separation of electron and hole confinements in the epitaxial growth direction. Devices using this system, such as the fabrication of long wavelength sources and detectors for fibre-optics communications, photodiodes, photocathodes, light-emitting diodes and double-heterostructure lasers have been successfully developed [5, 6]. Besides its technological potential, it also serves as a model system for investigating the atomic ordering and compositional modulation expected in III- $\mathrm{V}_{\mathrm{A}}-\mathrm{V}_{\mathrm{B}}$ alloys [7]. In spite of its importance, it has been noticed that there are some difficulties in growing high-quality samples [8, 9]. The specific difficulties in molecular beam epitaxy (MBE) of this structure arise as no unity sticking coefficients of group V species complicates the precise composition control of GaAsSb layer and excess amounts of $\mathrm{As}$ or $\mathrm{Sb}$ are still present on the surface even after As or Sb fluxes are closed. This makes control of abrupt interfaces difficult due to incorporation of an uncommon group V element, $\mathrm{Sb}$. The atomic configuration of these heterostructures at the interfaces can influence the optical and electronic properties of the devices due to changes in a quantum well profile.

In the present study, the effects of growth interruption at interfaces combined with $\mathrm{Sb}$ exposure of $\mathrm{GaAsSb} / \mathrm{GaAs}$ multiple quantum wells (MQWs) have been characterized by using phototransmittance (PT), contactless electroreflectance (CER) and wavelength modulated surface photovoltage spectroscopy (WMSPS). The red-shifts of the interband transitions are observed from samples grown under $\mathrm{Sb}$ exposure compared to the sample grown without interruption. An additional feature has also been observed below the GaAs region in $\mathrm{Sb}$ exposure-treated samples. The possible origin of the red shifts and additional feature are discussed.

\section{Experimental details}

The three GaAsSb/GaAs MQW samples employed in this study were grown on semi-insulating GaAs(100) substrates using a VG V-80MKII molecular beam epitaxy (MBE) apparatus. The $\mathrm{Sb}_{1}$ source was supplied using an EPI model 175 standard cracker K cell, and the $\mathrm{As}_{2}$ source was supplied from a Riber VAC 500 As valve cracker. The cracker zone temperature was $1050^{\circ} \mathrm{C}$, while the bulk zone temperature was about $430^{\circ} \mathrm{C}$. The structure of MQWs contains a $500 \mathrm{~nm}$ GaAs buffer layer and five periods of GaAsSb $6 \mathrm{~nm} / \mathrm{GaAs} 34 \mathrm{~nm}$. The growth interruption of GaAsSb/GaAs MQWs proceeded as follows. During growth, the cell shutter of Ga was kept open as long as necessary for the growth of the intended thickness of individual layers, while $\mathrm{Sb}$ termination was provided at the well/barrier interfaces. The interface exposure times were 10 and $30 \mathrm{~s}$ for samples II and III, respectively. In order to study the influence of $\mathrm{Sb}$ treatments, a specimen (sample I) was also prepared without any interruption. The samples were terminated with a $100 \mathrm{~nm}$ thick GaAs cap layer and all the layers were unintentionally doped. The growth conditions of these three samples were identical except for the Sb exposure times. The antimony composition in the GaAsSb layers was determined to be $0.18,0.20$ and 0.21 for samples I, II and III, respectively, by double crystal x-ray diffraction (DXRD) measurements.

PT measurement was achieved using an internally modulated $5 \mathrm{~mW}$ laser diode $(670 \mathrm{~nm})$ as the modulating source. The laser intensity was reduced to about $1-10 \%$ of its initial value by using a neutral density filter. The probe beam was incident through the front wire grid. A $150 \mathrm{~W}$ tungsten-halogen lamp filtered by a PTI $0.25 \mathrm{~m}$ monochromator provided the monochromatic light. The transmitted light was detected by an InGaAs photodetector placed behind the sample, and the signal was recorded by a lock-in amplifier. The DC output of the photodetector was maintained constant by a servo mechanism of a variable neutral density filter. For CER, an AC modulating voltage $(\sim 1 \mathrm{kV}$ at $200 \mathrm{~Hz})$ was applied between a front wire grid electrode and a 


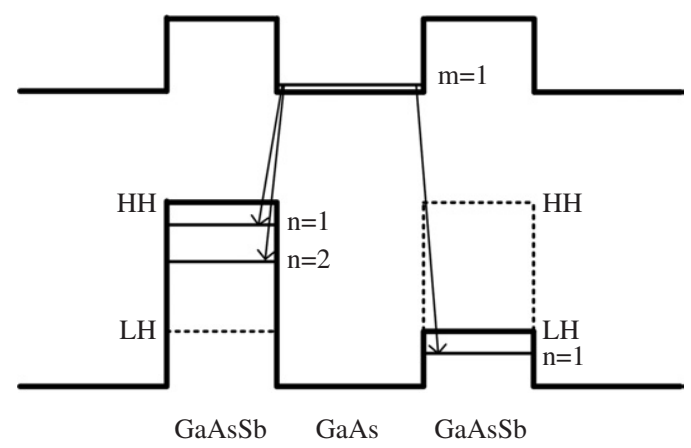

Figure 1. The schematic diagram of the band edges versus growth direction (without band bending) for a compressively strained GaAsSb/GaAs MQW structure, where the QW with the relevant heavyhole and light-hole transitions is indicated.

second electrode consisting of a metal plate [10]. These two electrodes were separated by an insulating spacing which provided a very thin layer $(0.1 \mathrm{~mm})$ of air between the front surface of the sample and the front electrode. The configuration is nondestructive as there is no direct contact with the front surface of the sample. The reflected light was detected by a silicon photodiode detector and an InGaAs photodetector for the visible and near infrared region, respectively. For WMSPS, the derivative-like surface photovoltage was measured between the sample and a reference metal grid electrode in a capacitive manner as a function of the photon energy of the probe beam with a wavelength-modulation technique. The illumination system consisted of a $150 \mathrm{~W}$ quartz-halogen lamp and a grating monochromator equipped with a vibrating exit slit operated by a power amplifier, employing a 2 inch loudspeaker as the transducer. A beam splitter was placed in the path of the incident light. The intensity of this radiation was monitored by a power meter and was kept constant by a stepping motor connected to a variable neutral density filter, which was also placed in the path of the incident beam. The incident light intensity was maintained at a constant level of $\sim 10^{-5} \mathrm{~W} \mathrm{~cm}^{-2}$. The illumination intensity and the amplitude of wavelength modulation were experimentally selected at levels not affecting the measured spectra; typically $\Delta \lambda / \lambda$ was of the order of $10^{-3}$. Precautions were taken to eliminate the spurious signals from light-intensity modulation. The normalized wavelength-modulated derivative-like photovoltage spectrum on the metal grid was measured with a copper plate as the ground electrode, using a buffer circuit and a lock-in amplifier.

\section{Results and discussion}

Figure 1 shows a schematic diagram of the band edges versus growth direction for a compressively strained GaAsSb/GaAs MQW structure. In contrast to the type-I semiconductor system, spatially separated electrons and holes are easily realized in such a type-II system, in which electrons are confined in the GaAs layer and holes are localized in the GaAsSb layer. The indirect transitions due to the spatially separated electrons and holes are restricted in a very narrow region near the interfaces.

The PT spectra in the region of $1.05-1.35 \mathrm{eV}$ at $300 \mathrm{~K}$ from the three GaAsSb/GaAs MQW samples are shown by the full lines in figure 2. It is noticed that the amplitude of the PT spectra of GaAsSb/GaAs MQWs is much smaller than that of InGaAs/GaAs quantum wells [13]. A probable reason is that the electrons and holes may not be confined in the same layer for a typeII structure. The open circles in figure 1 are least squares fits to the first-derivative Lorentzian 


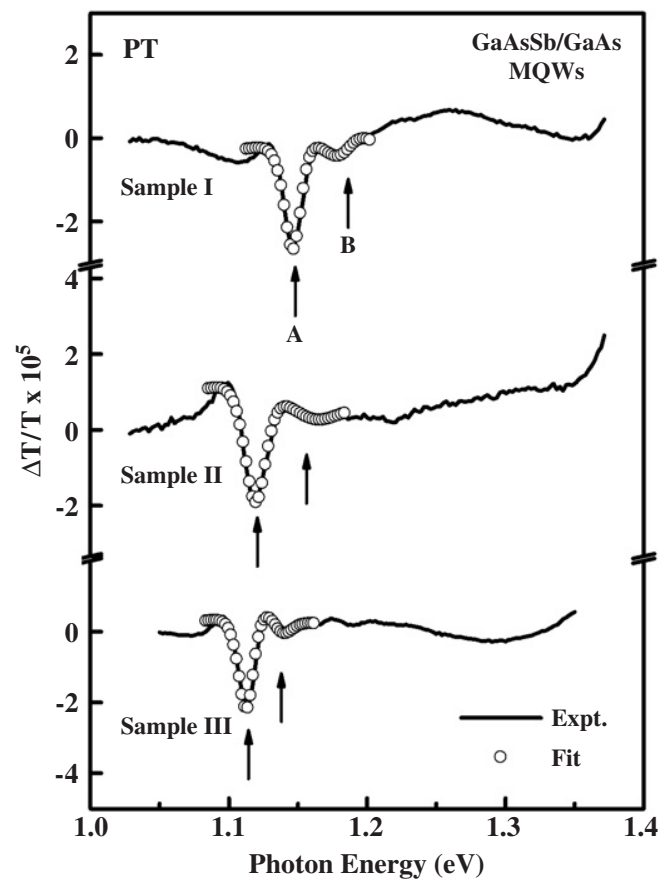

Figure 2. The PT spectra (full lines) from three $\mathrm{GaAs}_{1-x} \mathrm{Sb}_{x} / \mathrm{GaAs}$ samples taken at $300 \mathrm{~K}$. The open circles are the least-squares fits to the FDLL lineshape function. The obtained energy values are denoted by arrows.

lineshape (FDLL) function of the form $[11,12]$

$$
\frac{\Delta T}{T}=\operatorname{Re} \sum_{j=1} A_{j} \mathrm{e}^{\mathrm{i} \Phi_{j}}\left(E-E_{j}+\mathrm{i} \Gamma_{j}\right)^{-n}
$$

where $A_{j}$ and $\Phi_{j}$ are the amplitude and phase of the line shape, $E_{j}$ and $\Gamma_{j}$ are the energy and broadening parameter of the excitonic transitions, and the value of $n$ depends on the origin of the transitions. For the first derivative functional form, $n=2$ is appropriate for the bound states such as excitons $[11,12]$. The obtained interband energies are denoted by the letters $\mathbf{A}$ and $\mathbf{B}$ which correspond, respectively, to $11 \mathrm{H}$ (first electron to first heavy-hole) and $12 \mathrm{H}$ (first electron to second heavy-hole), and their respective locations are marked by arrows below the features and listed in table 1. It is obvious that these two quantum-well related features shift toward lower energies with the increase of the interruption time ( $\mathrm{Sb}$ exposure time). The observed red-shifts for Sb-treated samples can be understood to be due to increased bandalignment sensitivity toward the heterointerface state of these structures. Interface grading modifies the confining potential and involves complicated strain relief relaxation in the well. It has been demonstrated that in the heterostructures for which both group III and group V species differ in sublayers $[14,15]$, local strain is present at the interfaces. This may lead to modifications of the band lineup due to the contribution of interface dipoles formed by the bond length variations along heterointerfaces such as $\mathrm{Ga}-\mathrm{Sb}$ or $\mathrm{Ga}-\mathrm{As}$. The formation of the intermixed layers will further modify the actual band alignment through electron and hole confinements at the interfaces. Thus, the width and composition of the intermixed layers at the interfaces will influence the position and the linewidth of the interband transition features of the PT spectra. Compositional variations along the heterointerfaces as well as variations 


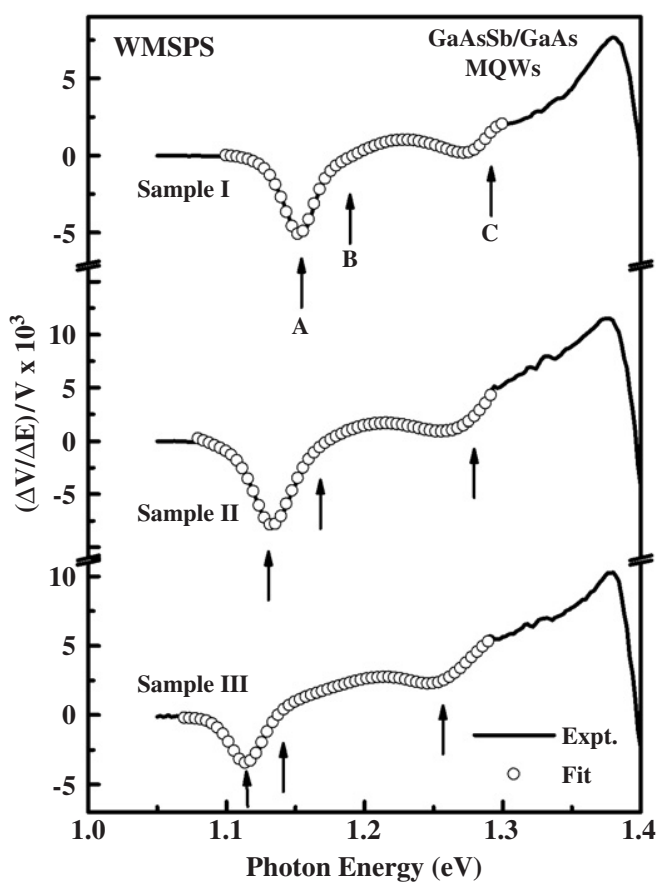

Figure 3. The WMSPS spectra (full lines) from three $\mathrm{GaAs}_{1-x} \mathrm{Sb}_{x} / \mathrm{GaAs}$ samples taken at $300 \mathrm{~K}$. The open circles are the least-squares fits to the FDLL lineshape function. The obtained energy values are denoted by arrows.

Table 1. Experimental and theoretical calculated values of the various interband transition energies of $\mathrm{GaAs}_{1-x} \mathrm{Sb}_{x} / \mathrm{GaAs} \mathrm{MQWs}$ at $300 \mathrm{~K}$.

\begin{tabular}{|c|c|c|c|c|c|c|c|}
\hline \multirow{2}{*}{$\begin{array}{l}\mathrm{GaAs}_{1-x} \mathrm{Sb}_{x} / \mathrm{GaAs} \\
\text { MQWs }\end{array}$} & & \multicolumn{3}{|c|}{$\begin{array}{l}\text { Experiment } \\
E_{m n \mathrm{H}(\mathrm{L})}(\mathrm{eV})\end{array}$} & \multicolumn{3}{|c|}{$\begin{array}{c}\text { Theory } \\
E_{m n \mathrm{H}(\mathrm{L})}(\mathrm{eV})\end{array}$} \\
\hline & & $11 \mathrm{H}$ & $12 \mathrm{H}$ & $11 \mathrm{~L}$ & $11 \mathrm{H}$ & $12 \mathrm{H}$ & $11 \mathrm{~L}$ \\
\hline Sample I & PT & $1.154 \pm 0.003$ & $1.188 \pm 0.005$ & & \multirow{2}{*}{1.150} & \multirow{2}{*}{1.196} & \multirow{2}{*}{1.287} \\
\hline$x=0.18$ & WMSPS & $1.154 \pm 0.003$ & $1.189 \pm 0.005$ & $1.291 \pm 0.005$ & & & \\
\hline Sample II & PT & $1.130 \pm 0.003$ & $1.161 \pm 0.005$ & & \multirow{2}{*}{1.122} & \multirow{2}{*}{1.169} & \multirow{2}{*}{1.262} \\
\hline$x=0.20$ & WMSPS & $1.129 \pm 0.003$ & $1.165 \pm 0.005$ & $1.280 \pm 0.005$ & & & \\
\hline Sample III & PT & $1.113 \pm 0.003$ & $1.138 \pm 0.005$ & & \multirow{2}{*}{1.109} & \multirow{2}{*}{1.157} & \multirow{2}{*}{1.254} \\
\hline$x=0.21$ & WMSPS & $1.115 \pm 0.003$ & $1.140 \pm 0.005$ & $1.256 \pm 0.005$ & & & \\
\hline
\end{tabular}

in size of the intermixed areas may be responsible for the linewidth increase observed in the samples with the $\mathrm{Sb}$ exposure treatment.

Figure 3 shows the WMSPS spectra of the three samples at room temperature. The open circles are the least-squares fits to the FDLL function $[11,12]$. Note that the spectra on the lower energy sides are asymmetric. In order to have a reasonable fit for these regions two interband transitions are needed. The obtained energies are designated by arrows. The fitted values are summarized in table 1 . The transition energies red-shifted with the increase of $\mathrm{Sb}$ exposure time. In contrast to the PT spectra, the WMSPS spectra contain an additional broad feature, denoted by $\mathbf{C}$, located at about $140 \pm 10 \mathrm{meV}$ above the $11 \mathrm{H}$ transitions. In general, we 
would expect PT to be the more sensitive of these two techniques. However, this is not the case in the present study. One possible reason might be due to the different modulation mechanism involved. PT is a form of electromodulation spectroscopy where the modulation of the electric field in the sample is caused by the photo-excited electron-hole pairs created by the laser diode. In type-II alignment, with the positive charges of the holes confined in the well region and the electrons experiencing electrostatic attraction toward the well region, it is difficult to achieve electric field modulation effectively in such an indirect transition configuration. Therefore PT becomes less sensitive compared to WMSPS in the study of type-II structures. The origin of feature $\boldsymbol{C}$ has been assigned as the first electron state to a light-hole transition and will be discussed in the following section.

Dumitras and Riechert have reported observing a feature located at $78 \mathrm{meV}$ above the PL peak in the derivative SPV spectrum of a type-II GaAs $/ \mathrm{GaAs}_{0.7} \mathrm{Sb}_{0.3} / \mathrm{GaAs}$ single quantum well [16]. The origin of this feature was identified as related to transitions between the quantum well localized hole state and the extended electron states. By comparing the energy difference of their observed features with that of our observation and the fact that the present samples have lower $\mathrm{Sb}$ contents, we can tentatively rule out the assignment for the currently observed feature. In order to specify the observed spectral features, we have calculated the confined energy levels in both the conduction and valence bands by assuming abrupt interfaces. We have solved a onedimensional Schrödinger equation for five finite square quantum wells based on the envelope function approximation [17] including the effects of strain [18]. A value of unstrained valance band band-offset $Q_{\mathrm{v}}^{0}=0.85$ was used in the calculation [19]. We have used a number of relevant parameters of $\mathrm{GaAs}_{1-x} \mathrm{Sb}_{x}$ listed in [8]. The effective masses of electron, heavyhole and light-hole, the lattice constant, hydrostatic $(a)$ and shear $(b)$ deformation potentials, and the elastic stiffness constants $C_{11}$ and $C_{12}$ of the ternary material were obtained by linear interpolation of values of the end-point semiconductors GaAs and GaSb [20]. The calculated results are listed in table 1 . A reasonable agreement between experiments and theoretical calculations can be achieved if we identify the features $\mathbf{A}, \mathbf{B}$ and $\mathbf{C}$ to be $11 \mathrm{H}, 12 \mathrm{H}$ and $11 \mathrm{~L}$, respectively. It is noticed that the separation between $11 \mathrm{H}$ and $12 \mathrm{H}$ transitions determined from PT/WMSPS reduces as the Sb exposure time increases. This is different from the theoretical prediction that as the $\mathrm{Sb}$ content increases the separation should become slightly larger. Our observation to the contrary might be due to the intermixing effect at the interfaces and results in a change of the shape of quantum well profiles and modifies transition energies.

Figure 4 shows CER spectra of the samples in the region of 1.3-1.55 eV. The CER spectra exhibit Franz-Keldysh oscillations (FKOs) above the band edge of GaAs. The period of the FKOs is a direct measure of the built-in electric field at the interface of GaAsSb/GaAs [11, 12]. The built-in electric field at the GaAsSb/GaAs interfaces is increased with the interruption time. An additional feature located below GaAs band edge, denoted as $\mathbf{X}$, is observed on the samples under $\mathrm{Sb}$ exposure treatment. The probable origin of this feature will be discussed in the following section.

Figure 5 shows the DC optical bias dependent CER spectra of sample III in the vicinity of the GaAs region with an additional steady-state illumination of a $670 \mathrm{~nm}$ laser diode as an optical bias. From the CER spectra, FKOs above the GaAs band edge were observed. In contrast to most of the cases, the built-in electric field determined from the period of the FKOs increases slightly with the intensity of the DC laser bias. The increase of the built-in electric field with the intensity of the DC laser can be understood as follows. As shown in figure 1 the energy minima for electrons and holes lie in different layers. This means that spatially separated electrons and holes are easily realized in such a system, in which electrons are confined in the GaAs layer and holes are localized in the GaAsSb layer. Due to the appearance of the interface states as a consequence of $\mathrm{Sb}$ absorption at the interface and/or the possible interchanging 


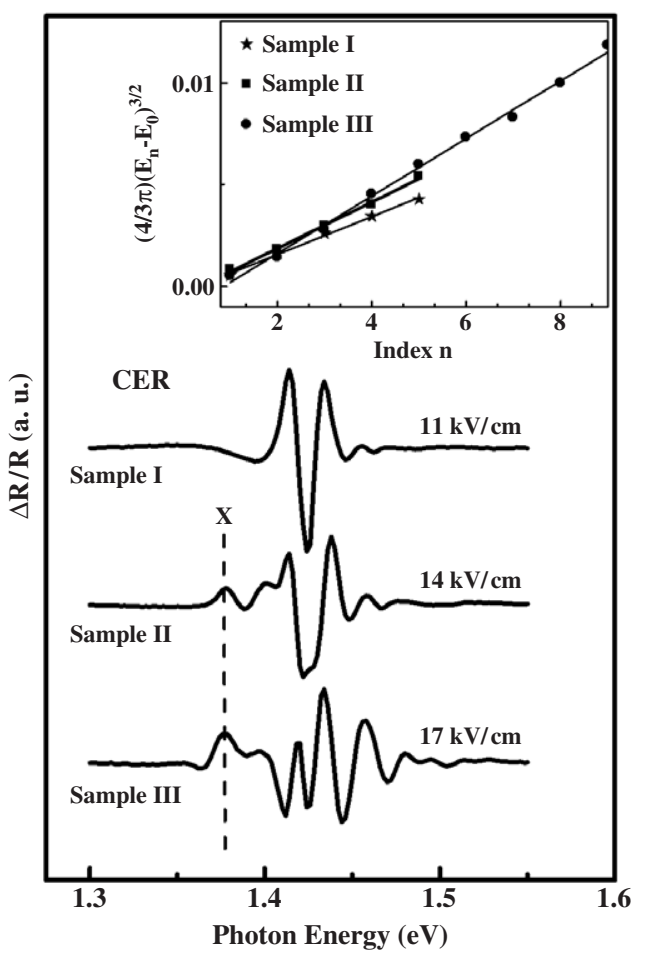

Figure 4. The CER spectra of three samples in the range from $1.3-1.55 \mathrm{eV}$. An additional feature is observed on samples under $\mathrm{Sb}$ exposure treatment. The inset shows plots of $(4 / 3 \pi)\left(E_{n}-E_{0}\right)^{3 / 2}$ as a function of the index $n$ which labels the extrema.

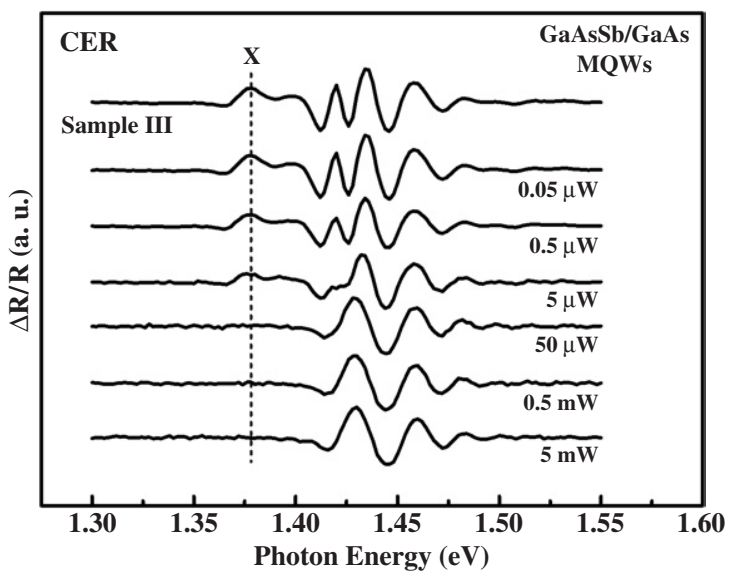

Figure 5. The DC optical bias dependent CER spectra of sample III in the vicinity of the GaAs region. The built-in electric field increases slightly and the additional feature disappeared for DC bias exceeding $50 \mu \mathrm{W}$.

reaction between the $\mathrm{Sb}$ atoms and the As atoms, it is difficult to grow GaAsSb/GaAs MQWs with sharp interfaces. Therefore, in the absence of DC optical bias we always have a slight band bending for the type-II structures (see figure 6). Along with the DC laser illumination, the 


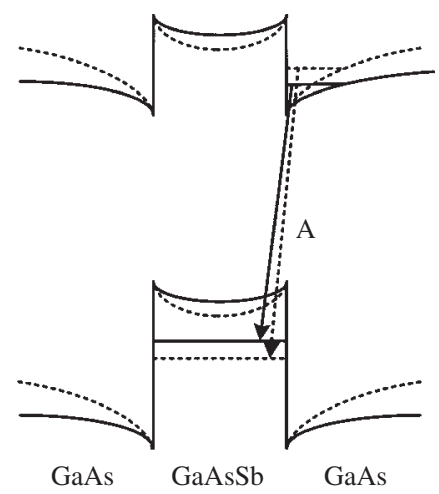

Figure 6. The schematic energy band alignment with band bending of GaAsSb/GaAs MQWs: solid lines (absence of DC optical bias); dotted lines (with DC optical bias).

photo-generated electrons can move across the GaAsSb/GaAs interface toward the GaAs layer region, leaving behind holes in the GaAsSb region. A direct consequence of this electron-hole separation is an increase in the band bending (as shown by dotted lines in figure 6) and the built-in electric field at the interface. The increase in band bending shifted the localized states in the conduction band upward. As a result the $11 \mathrm{H}$ transition blue-shifted.

As shown in figure 5, without DC laser bias a feature denoted as $\mathbf{X}$ located around $1.375 \mathrm{eV}$ is clearly visible. The intensity of this feature gradually decreases with increasing illumination of the optical bias and can be eliminated completely for optical bias exceeding $50 \mu \mathrm{W}$. The same phenomenon is also being observed in sample II. The additional feature is only observed on the samples under $\mathrm{Sb}$ exposure treatment and its location is lower than that of GaAs impurity levels [21], thus ruling out the possibility that it belongs to a sub-bandgap feature of GaAs. In this study, we have tentatively assumed the feature to originated from the transition between the valence band of the GaAs barrier to the localized interface states due to the presence of Sb. Our tentative assertion is based on the observation of the complete elimination of this feature for optical bias exceeding $50 \mu \mathrm{W}$. The steady-state optical illumination of the sample may result in a completely filled interface states as the power exceeds $50 \mu \mathrm{W}$. When the interface states are completely occupied, the transition between the valence band of the GaAs barrier and the interface states is prohibited, resulting in the disappearance of the feature in the optically biased CER spectra. However, the true origin of this feature warrants further verification, and more studies are required.

\section{Summary}

In summary we report a modulation spectroscopy study of GaAsSb/GaAs MQWs with different interface treatments. The results indicate that $\mathrm{Sb}$ exposure growth interruptions change significantly the state of the interfaces and can thus influence the optical response. The red-shift of the observed features and a broader lineshape of the fundamental transition are observed with increasing interface exposure time. The results may be due to an increase of the Sb content and intermixing of $\mathrm{Sb}$ at the GaAs interface layers in the samples. From the CER spectra, we have observed an additional feature below the GaAs region in $\mathrm{Sb}$ exposure samples. This feature disappeared for DC bias exceeding $50 \mu \mathrm{W}$. The physical origin of the feature is tentatively attributed to the transition between the valence band of the GaAs barrier and the localized interface states due to the presence of $\mathrm{Sb}$. 


\section{Acknowledgment}

The authors acknowledge the financial support from National Science Council of Taiwan under project no NSC94-2215-E-011-002.

\section{References}

[1] Kondow M, Kitatani T, Aoki M, Nakatsuka S and Kudo M 2001 LEOS 2001: Proc. 14th Annual Meet. IEEE Lasers \& Electro-Optics Society (San Diego, 2001) (Piscataway, NJ: IEEE) p 622

[2] Liu H Y, Steer M J, Badcock T J, Mowbray D J, Skolnick M S, Navaretti P, Groom K M, Hopkinson M and Hogg R A 2005 Appl. Phys. Lett. 86143108

[3] Liu P W, Liao G H and Lin H H 2002 Electron. Lett. 381354

[4] Quochi F, Kilper D C, Cunningham J E, Dinu M and Shah J 2001 IEEE Photon. Technol. Lett. 13921

[5] Dvorak M W, Bolognesi C R, Pitts O J and Watkins S P 2001 IEEE Electron. Device Lett. 22361

[6] Sun X, Hsu J, Zheng X G, Campbell J C and Holmes A L 2002 IEEE Photon. Technol. Lett. 14681

[7] Yano M, Ashida M, Kawaguchi A, Iwai Y and Inoue M 1989 J. Vac. Sci. Technol. B 7199

[8] Chiu Y S, Ya M H, Su W S and Chen Y F 2002 J. Appl. Phys. 925810

[9] Liu G, Chuang S L and Park S H 2000 J. Appl. Phys. 885554

[10] Huang Y S and Pollak F H 2005 Phys. Status Solidi a 2021193

[11] Aspnes D E 1980 Handbook on Semiconductor vol 2, ed T S Moss (New York: North-Holland) p 109

[12] Pollak F H and Shen H 1993 Mater. Sci. Eng. R 10275

[13] Hsu H P, Huang Y S and Tiong K K 2006 unpublished

[14] Hybertsen M S 1990 Phys. Rev. Lett. 641984

[15] Nelson J S, Kurtz S R, Dawson L R and Lott J A 1990 Appl. Phys. Lett. 57578

[16] Dumitras Gh and Riechert H 2003 J. Appl. Phys. 943955

[17] Bastard G 1981 Phys. Rev. B 245693

[18] Chuang S L 1995 Physics of Optoelectronic Devices (New York: Wiley) p 144

[19] Chen T T, Chen C H, Cheng W Z, Su W S, Ya M H, Chen Y F, Liu P W and Lin H H 2003 J. Appl. Phys. 939655

[20] Hellwege K H (ed) 1982 Numerical Data and Functional Relationships in Science and Technology (LandoltBornstein New Series Group III vol 17a) (Berlin: Springer)

Hellwege K H (ed) 1982 Numerical Data and Functional Relationships in Science and Technology (LandoltBornstein New Series Groups III-V vol 22a) (Berlin: Springer)

[21] Tober R L, Pamulapati J, Oh J E and Bhattacharya P K 1990 J. Appl. Phys. 686388 